\title{
Measuring voltage transients with an ultrafast scanning tunneling microscope
}

Keil, Ulrich Dieter Felix; Jensen, Jacob Riis; Hvam, Jørn Märcher

Published in:

Applied Physics Letters

Link to article, DOI:

10.1063/1.118938

Publication date:

1997

Document Version

Publisher's PDF, also known as Version of record

Link back to DTU Orbit

Citation (APA):

Keil, U. D. F., Jensen, J. R., \& Hvam, J. M. (1997). Measuring voltage transients with an ultrafast scanning tunneling microscope. Applied Physics Letters, 70(19), 2625-2627. https://doi.org/10.1063/1.118938

\section{General rights}

Copyright and moral rights for the publications made accessible in the public portal are retained by the authors and/or other copyright owners and it is a condition of accessing publications that users recognise and abide by the legal requirements associated with these rights.

- Users may download and print one copy of any publication from the public portal for the purpose of private study or research.

- You may not further distribute the material or use it for any profit-making activity or commercial gain

- You may freely distribute the URL identifying the publication in the public portal

If you believe that this document breaches copyright please contact us providing details, and we will remove access to the work immediately and investigate your claim. 


\title{
Measuring voltage transients with an ultrafast scanning tunneling microscope
}

\author{
Ulrich D. Keil, ${ }^{a}$ Jacob R. Jensen, and Jørn M. Hvam \\ Mikroelektronik Centret, DK-2800 Lyngby, Denmark
}

(Received 4 December 1996; accepted for publication 11 March 1997)

\begin{abstract}
We use an ultrafast scanning tunneling microscope to resolve propagating voltage transients in space and time. We demonstrate that the previously observed dependence of the transient signal amplitude on the tunneling resistance was only caused by the electrical sampling circuit. With a modified circuit, where the tunneling tip is directly connected to the current amplifier of the scanning tunneling microscope, this dependence is eliminated. All results can be explained with coupling through the geometrical capacitance of the tip-electrode junction. By illuminating the current-gating photoconductive switch with a rigidly attached fiber, the probe is scanned without changing the probe characteristics. (c) 1997 American Institute of Physics.
\end{abstract}

[S0003-6951(97)00419-1]

Ultrafast probes for measuring picosecond and subpicosecond electrical devices were thus far based on electro-optic sampling and photoconductive (PC) sampling. ${ }^{1}$ Electro-optic sampling probes show the highest temporal resolution (down to $200 \mathrm{fs}$ ), but spatial resolution is limited to about $3 \mu \mathrm{m}$. In contrast to PC sampling, electro-optic sampling does not require electrical contact to the device under test. It is only recently that $\mathrm{PC}$ sampling has been extended to noncontact measurements. ${ }^{2}$ PC sampling has in general a higher sensitivity but a lower time resolution than electro-optic sampling. The spatial resolution of PC probes has not been an issue yet.

Based on the PC sampling technique, another approach has been introduced by Weiss et $\mathrm{al}^{3}{ }^{3}$ They reported picosecond and subpicosecond ${ }^{4}$ time resolution with a photoconductively gated scanning tunneling microscope. Their results have been confirmed by two other groups. ${ }^{5-7}$ The spatial resolution is still subject to controversy, ${ }^{8}$ and simultaneous spatial and temporal resolution has so far not been demonstrated directly. A linear dependence of the amplitude on the dc tunneling current or gap conductance has been observed consistently and signal shapes have varied from a derivative of the measurement with the tip in contact to signals more resembling the contact measurement.

Our instrument is based on the ultrafast scanning tunneling microscope (USTM) presented in Ref. 3 and details of our setup are reported in Ref. 6. The setup differs in two major aspects from previously reported designs. The tunneling tip is attached to a coplanar strip line (CPS) which provides direct electrical access to the tip for monitoring the dc tunneling current. Second, we attach a fiber directly to the PC switch which enables an unconstrained scanning of the tip without variations in the illumination of the PC switch. Both modifications help resolve the actual operation principle of the instrument: (1) The previously observed exponential distance dependence disappears by changing the electrical measurement circuit. (2) The time-resolved field of a propagating electrical wave can be mapped out by scanning across a transmission line.

The gated probe is attached to the piezo scanner tube of a commercial, ambient scanning tunneling microscope and

${ }^{\text {a)} E l e c t r o n i c ~ m a i l: ~ u l l i @ m i c . d t u . d k ~}$ the test sample is mounted above the tip. On the test sample, a subpicosecond electrical pulse is generated by illuminating an in-line PC switch on a coplanar waveguide (CPW) with a $100 \mathrm{fs}$ laser pulse. The electrical pulse is picked up by the gated tunneling tip. The use of a fiber enables us to perform scans in the surface plane with a range of $50 \mu \mathrm{m}$.

The test CPW has a $5 \mu \mathrm{m}$ contact spacing and contact width. All three lines at the position of the tunneling tip are kept at a bias voltage, $V_{b}$, of around $50 \mathrm{mV}$ to supply a dc tunneling current. The switch voltage, $V_{\text {sw }}$ (typically 2-10 V) is applied to the other side of the in-line switch. From electro-optic sampling ${ }^{9}$ measurements, we deduce that the pulse width of the input signal is $800 \mathrm{fs}$ and the amplitudes are between $100 \mathrm{mV}$ and $1 \mathrm{~V}$. Figure 1(a) shows the amplitudes of the time resolved signals measured with the USTM probe on the center electrode. First, the signal is measured in the tunneling regime with the tip distance being controlled through the dc tunneling current. In this range, the tunneling resistance, $R_{t}$, is varied from 1 to $170 \mathrm{M} \Omega$. An important result in this letter is that in contrast to previously reported measurements, the amplitude is independent of the tunneling resistance [Fig. 1(a)]. (In another set of measurements, we increased $R_{t}$ up to $1.3 \mathrm{G} \Omega$ without a notable change in the amplitude.) When the tip is retracted to a distance of $50 \mu \mathrm{m}$, the signal amplitude drops by a factor of approximately four. This amplitude dependence roughly resembles that of a geometrical capacitance formed by a sphere over an infinite conducting plane. A more realistic model has to take into account the limited width of the center conductor, the wire shape of the tip, and consider a different local radius in the tunneling regime.

As a test that the signal amplitude is determined by the modified setup, we disconnect the internal preamplifier in an out-of-range measurement. With the tip floating, the signal vanishes completely in the noise. As has been observed previously, the signal shape in Fig. 1(b) is resembling a derivative of the propagating voltage pulse (see discussion of Fig. 3). ${ }^{3-7}$ Figure 1(b) also shows that the signal shape changes when the tip is retracted out of the tunneling regime. The total signal in the tunneling regime appears to be consist of two components. The component (at $\tau=9 \mathrm{ps)}$ disappears outside of the tunneling regime and a component with a 

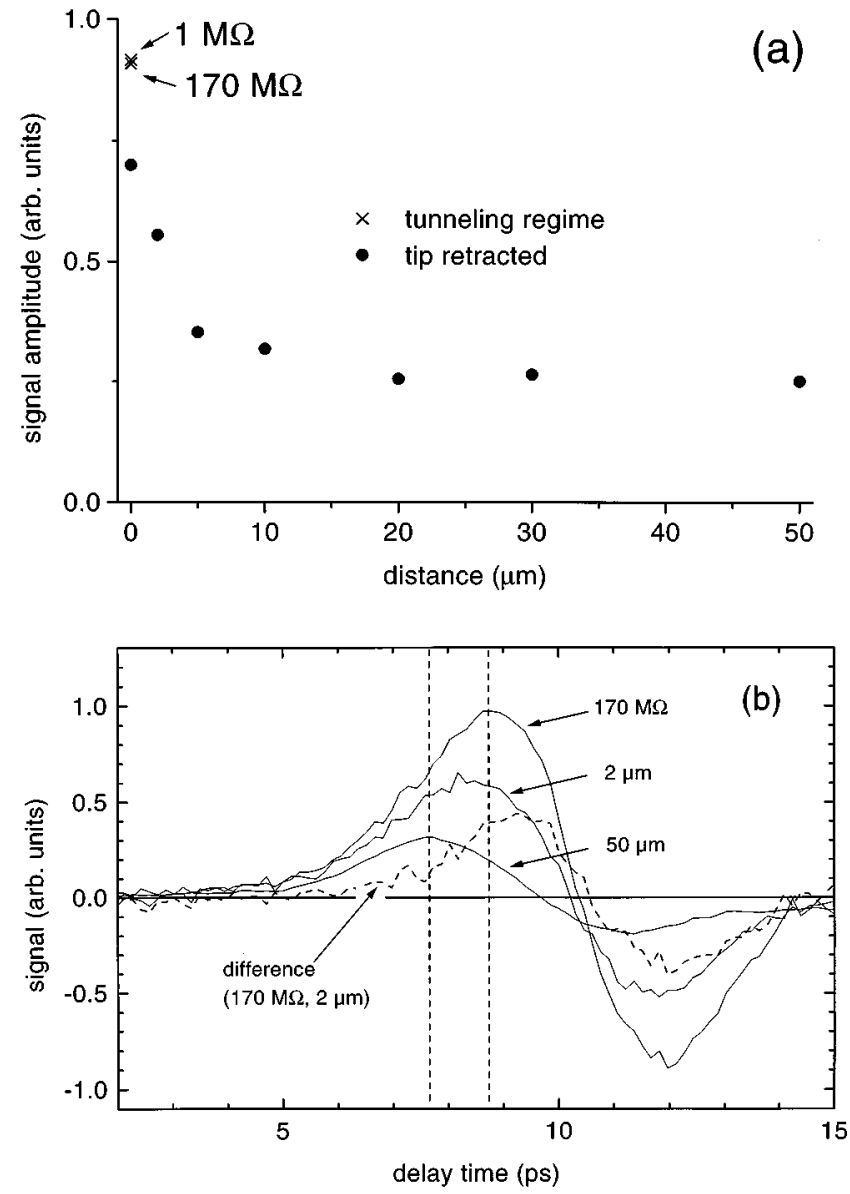

FIG. 1. (a) Dependence of the transient signal amplitude on the dc tunneling resistance in tunneling mode and out of tunneling range for different distances. (b) Transient signals for a $170 \mathrm{M} \Omega$ tunneling resistance and different distances. In addition, the difference between the $170 \mathrm{M} \Omega$ measurement and the measurement at $2 \mu \mathrm{m}$ is shown. The vertical lines indicate the positions of the maxima for the $170 \mathrm{M} \Omega$ and the $50 \mu \mathrm{m}$ measurement.

broader response in time remains. Comparing the measurement at $2 \mu \mathrm{m}$ tip electrode distance with the measurement in the tunneling regime $(170 \mathrm{M} \Omega)$, we observe the following: First $(\tau<7.8 \mathrm{ps})$, the signals are almost identical. We assign this part of the signal to the extended field from the CPW electrodes to the full length of the $200 \mu \mathrm{m}$ tip wire which is only marginally influenced by retracting the tip by $2 \mu \mathrm{m}$. For longer delay times ( $\tau>8 \mathrm{ps})$ the transients start to differ as illustrated by the difference shown in Fig. 1(b). This component in the tunneling measurement we attribute to the capacitive contribution in the vicinity of the tunneling region. When we retract the tip to a distance of $50 \mu \mathrm{m}$, only the leading signal component remains. The maximum shifts to a shorter delay time and broadens compared to the tunneling measurement. The broader and earlier temporal response is consistent with the geometrical capacitance picture. For a longer distance, the field lines spread over a larger area and are thus influenced by the propagating voltage pulse for a longer time. In the tunneling regime, the more confined field of the tunneling junction and the extended field from the full length of the $200 \mu \mathrm{m}$ tip wire to the CPW electrodes contribute to the signal. For the signal to be picked up in the tunneling region, the voltage pulse has to propagate on the transmission line to the tunneling junction and is therefore

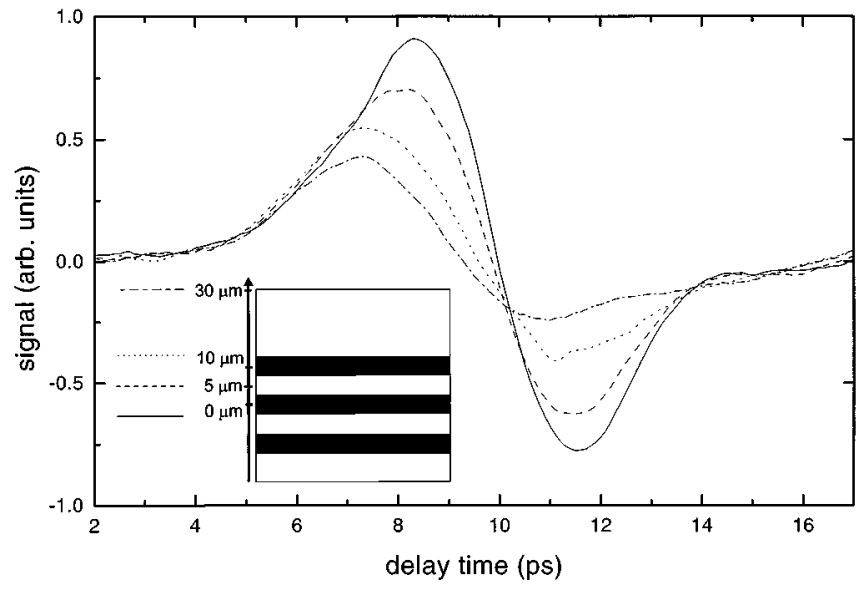

FIG. 2. Transient signals at different selected positions. The tip positions with respect to the CPW are shown in the inset.

delayed with respect to the long range signal.

These two components in the signal in Fig. 1(b) can also be resolved in the measurement shown in Fig. 2. Here the tip is stepped across the CPW while being in the tunneling regime on top of the electrodes. On the insulating semiconductor substrate, a tunneling current cannot be drawn and the tip is in direct (physical) contact with the substrate. At each position, a scan in delay time is carried out. The position of the transmission line is measured by monitoring the dc tunneling current which is only measurable on the gold transmission lines. Figure 2 shows the transients for four selected positions on the CPW. The result shows that the high amplitude component [at $\tau=8.5 \mathrm{ps}$ in Fig. 2, at $\tau=9 \mathrm{ps}$ in Fig. $1(b)]$ is only observed on the center electrode. In contrast, the broad, leading signal component is observed everywhere in the scanning range. We attribute this observation to the pulse generation principle. By illuminating the in-line gap, the voltage on the center line is increased. This increase results in a transient voltage difference between the center electrode and the ground electrodes, the fundamental differential mode of the CPW. In addition, the average voltage of all the lines is increased, i.e., a common mode is excited together with the differential mode. For the common mode, the CPW can be seen as a single wire with the field lines spreading out to an external ground. Due to the lack of a defined ground electrode, the field is expected to be spatially extended. We have compared these measurement to a pulse generated between the transmission line where only a differential mode is excited. The results show a better confinement of the electrical wave in this case and a common mode signal is not observed. The details of these measurements will be presented elsewhere. ${ }^{10}$ We point out that the distinction between common and differential mode is generally not considered in pulsed PC or electro-optic sampling measurements. The existence of a common mode can neither be detected by PC sampling of a single electrode nor in electro-optic sampling configurations where the field between the transmission line electrodes is measured. In the literature about (sub)picosecond pulse propagation, to our knowledge, only the differential, fundamental mode of transmission lines has been considered so far. In a more general transmission line theory, all modes have to be taken into account. ${ }^{11}$ 

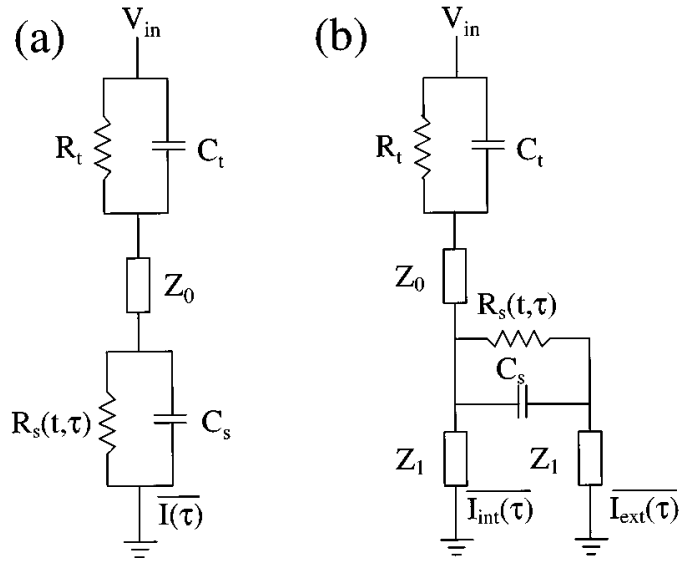

FIG. 3. Equivalent circuits: (a) unmodified floating tip setup, (b) modified setup with two current preamplifiers.

With the equivalent circuit shown in Fig. 3, we point out some experimental details specific for our setup. For a closely spaced tunneling junction and PC switch as in Ref. 5, junction and switch can be regarded as lumped elements. In our case, the time resolution of about $2 \mathrm{ps}$ is smaller than the propagation time of approximately 3 ps (for a total distance of $300 \mu \mathrm{m}$ ) between junction and PC switch. In this case, the characteristic impedance of the tip wire (and the CPS), $Z_{0}$, has to be considered. ${ }^{12} Z_{0}$ represents a combined characteristic impedance of the tip wire and the CPS in front of the PC switch. If the capacitive contribution at the tunneling junction is dominating, then $C_{1}$ and $Z_{0}$ form a differentiator. The transitions from tunneling junction to wire, and wire to CPS, both form impedance mismatches resulting in attenuation and reflection of the signal. ${ }^{7}$ For the time resolved signal, only the propagation path to the PC switch is important. If reflections from the end of the transmission line are disregarded, the signal path behind the PC switch serves merely for the measurement of delay time dependent dc signals. The two lines of this section of the CPS are represented by the characteristic impedances, $Z_{1}$.

In our detection scheme, the internal amplifier, controlling the feedback loop, is connected to the tip directly through one strip of the transmission line and the external amplifier is connected through the PC switch. The decisive difference, compared to previously reported setups, is that the tip is connected to the virtual ground of the internal preamp and is, therefore, not floating. Qualitatively measurements with and without a floating tip are equivalent. ${ }^{7}$ Groeneveld et $a l .{ }^{9}$ have pointed out that the floating tip can explain the previously observed amplitude dependence. The implied exponential distance dependence is thus not indicating a high lateral spatial resolution. It must be kept in mind that in this sampling technique only a dc current is measured. The measured signals $I_{\text {int }}(\tau)$ or $I_{\text {ext }}(\tau)$ are averaged over real time, $t$, and depend only on the delay time, $\tau$. In the unmodified detection circuit, this dc current can only be drawn through $R_{t}$. By connecting a second pre-amp, an additional current path is possible. As can be seen from the equivalent circuit, the dc and ac component of the signal are not strictly separated. On the "tip line", the dc current is nearly unattenuated but is reduced when the PC switch is excited. On the other line, the dc current has to pass the gap resistance of the transmission line. This resistance of 5-20 M $\Omega$ can be kept small compared to the tunneling resistance but presents a limit for the minimum selectable tunneling resistance. When the PC switch is excited, the current increases on this line. The transient signal can thus be measured on both lines. The main reason for using two amplifiers is that the cut-off frequency of the external amplifier can be chosen higher than for the internal amplifier. The modulation frequencies were kept above the cut-off frequency of the feedback loop and below the cut-off frequency of the external preamp. By these means, the influence of the modulated signal on the feedback loop is minimized. When measuring the modulated, delay dependent signal with the internal preamp (while the tip is connected to virtual ground of the external preamp), we observe indeed the same signal as with the external preamp with the opposite sign. It should be noted that this mode of operation is almost equivalent to a recently reported noncontact PC sampling probe. ${ }^{2}$

In summary, we use a fiber coupled photoconductively gated STM to resolve propagating voltage transients in space and time. We demonstrate and use the ability to freely position the tip on the sample. By using fiber illumination of the probe $\mathrm{PC}$ switch we achieve an excellent reproducibility. The mode structure of a pulse propagating on a CPW can be resolved. Most importantly, by modifying the detection circuit, we do not observe an exponential distance dependence of the transient signal amplitude. The signal amplitude drops by a factor of four by retracting the tip out of the tunneling regime up to $50 \mu \mathrm{m}$ above the surface. The probe acts as a transient voltage probe coupled through the geometrical capacitance which consists of a localized contribution in the tunneling region and a distributed contribution formed by the full length of the tip wire and the transmission line electrodes.

The authors thank Claus Sørensen from the III-V Nanolab for the growth of the LT-GaAs samples and Ole Hansen for fruitful discussions. This work is supported by the Danish Research Academy through a DANVIS grant and by the Danish Ministries of Research and Industry in the framework of CNAST.

${ }^{1}$ J. Kim, S. Williamson, J. Nees, S. Wakana, and J. Whitaker, Appl. Phys. Lett. 62, 2268 (1993).

${ }^{2}$ J.-R. Hwang, R. K. Lai, J. Nees, T. Norris, and J. F. Whitaker, Appl. Phys. Lett. 69, 2211 (1996)

${ }^{3}$ S. Weiss, D. F. Ogletree, D. Botkin, M. Salmeron, and D. S. Chemla, Appl. Phys. Lett. 63, 2567 (1993).

${ }^{4}$ D. Botkin, J. Glass, D. S. Chemla, D. F. Ogletree, M. Salmeron, and S. Weiss, Appl. Phys. Lett. 69, 1321 (1996).

${ }^{5}$ R. H. M. Groeneveld, Th. Rasing, L. M. F. Kaufmann, E. Smalbrugge, J. H. Wolter, M. R. Melloch, and H. van Kempen, J. Vac. Sci. Technol. B 14, 861 (1996).

${ }^{6}$ U. D. Keil and J. M. Hvam, Technical digest, EQEC '96, Hamburg, Germany (unpublished).

${ }^{7}$ U. D. Keil, J. R. Jensen, and J. M. Hvam, J. Appl. Phys. (to be published).

${ }^{8}$ R. H. M. Groeneveld and H. van Kempen, Appl. Phys. Lett. 69, 2294 (1996)

${ }^{9}$ J. A. Valdmanis and G. Mourou, IEEE J. Quantum Electron. 22, 69 (1986).

${ }^{10}$ J. R. Jensen, U. D. Keil, and J. M. Hvam, Appl. Phys. Lett. (in press).

${ }^{11}$ A. A. Smith Jr., Coupling of External Electromagnetic Fields to Transmission Lines (Wiley, New York, 1977).

${ }^{12}$ D. A. Botkin, Ph.D. thesis, UC Berkeley, 1995. 